\title{
V3
}

\section{Distinguer les risques sanitaire et nutritionnel perçus pour améliorer les comportements alimentaires}

\author{
Marie-Eve Laporte \\ IAE Paris - Sorbonne Business School, laboratoire GREGOR \\ laporte.iae@univ-paris1.fr
}

\section{Résumé}

L'alimentation est étroitement liée au bien-être et à la santé. De ce fait, elle suscite aussi des inquiétudes. La présente recherche étudie la perception du risque alimentaire à travers ses deux dimensions théoriques: (1) la perception de risque sanitaire à court terme et (2) la perception de risque nutritionnel à long terme. Elle vérifie par deux études quantitatives en France que les consommateurs distinguent ces deux dimensions et que la seconde est désormais prépondérante. Le renforcement des normes pour lutter contre le risque sanitaire a paradoxalement créé une distance entre le consommateur et son assiette, accroissant la perception de risque nutritionnel. Il apparaît nécessaire de prendre celle-ci en compte pour rassurer les consommateurs, sans pour autant négliger le risque sanitaire. L'article analyse différentes solutions de le faire en pratique.

Mots-clés: alimentation ; nutrition ; risque perçu ; risque nutritionnel ; outils digitaux

\begin{abstract}
Distinguishing between perceived health and nutritional risks to improve eating behaviors

Food is closely associated with well-being and health. In return, it also causes concerns. The present research studies perception of food risk through its two dimensions: (1) perception of health risk in the short term and (2) perception of nutritional risk in the long term. Two quantitative studies verify in France that consumers distinguish both dimensions and that perception of nutritional risk is now prominent. Implementing tighter standards against health risk has paradoxically created a distance between consumers and their plate, increasing perception of nutritional risk. The latter should be taken into account to reassure consumers, without overlooking health risk. The present paper analyses practical tools to do so.
\end{abstract}

Keywords: Food; nutrition; perceived risk; nutritional risk; digital tools 


\section{Distinguer les risques sanitaire et nutritionnel perçus pour améliorer les comportements alimentaires}

\section{Introduction}

L'alimentation occupe une place à part dans la consommation des individus. En effet, elle contribue quotidiennement à leur bien-être et à leur santé. Parce qu'elle comporte une dimension de vie ou de mort, l'alimentation incarne « l'acte le plus fondamental de tout être humain et celui qui peut avoir le plus d'impact sur sa santé »(Bergadaà et Urien 2006). Elle suscite dès lors des sentiments ambigus, entre plaisir de manger et de partager son repas, et inquiétude pour sa santé. L'acte d'ingestion fait courir un risque physique à l'individu, celui d'incorporer des aliments dangereux pour sa santé, voire mortels. Pour cette raison, les peurs alimentaires ont vraisemblablement toujours existé, toutefois elles semblent prendre de l'ampleur aujourd'hui (Pichon 2012). Plusieurs enquêtes révèlent que les Français se soucient davantage des effets des aliments sur la santé ${ }^{1}$. De plus, la nature des peurs alimentaires semble évoluer. Alors qu'autrefois, les personnes redoutaient l'intoxication alimentaire, aujourd'hui elles craignent de développer des affections graves (Rozin 2005). On retrouve là les deux dimensions théoriques du risque alimentaire (Aurier et Sirieix 2009) : le risque sanitaire de tomber malade à court terme suite à l'ingestion de nourriture avariée, et le risque nutritionnel de détériorer sa santé à long terme par les effets secondaires d'une alimentation déséquilibrée.

En pratique, les consommateurs distinguent-ils les deux dimensions du risque alimentaire ? La perception du risque nutritionnel est-elle réellement plus élevée que la perception du risque sanitaire aujourd'hui? Auquel cas faut-il concentrer la communication sur le risque nutritionnel, et sous quelle forme ? Ces questions présentent de l'intérêt pour les politiques de santé publique et le marketing alimentaire. En complément d'autres mesures, une meilleure prise en compte des risques alimentaires perçus pourrait favoriser des comportements plus sains et contribuer à diminuer les affections nutritionnelles chroniques telles que les maladies

\footnotetext{
${ }^{1}$ D'après le Crédoc, en 2013, 87\% des Français considéraient que leur alimentation influençait leur santé, contre 75\% en 1997. D'après l'étude de Kantar pour Ethicity-Greenflex, en 2016, 89\% des Français déclaraient choisir leurs aliments pour rester en bonne santé. Dans le baromètre Santé Nutrition 2008 de l'Institut national de prévention et d'éducation pour la santé, les Français considéraient que l'alimentation jouait un rôle important dans l'apparition de l'obésité (97\%), des maladies cardio-vasculaires (95\%), du diabète (94\%), de l'ostéoporose $(85 \%)$ et du cancer $(72 \%)$.
} 
cardio-vasculaires, le diabète de type deux, l'obésité ou certains cancers. Elle permettrait aussi de contrer certaines pratiques alimentaires potentiellement dangereuses pour la santé des individus. Pour répondre aux interrogations soulevées, une revue de la littérature sur le lien historique entre alimentation et santé et sur le risque perçu est menée afin de clarifier le concept de perception du risque alimentaire. Les résultats de deux études quantitatives sont ensuite analysés pour établir si les propositions se vérifient empiriquement. Enfin, les implications en matière de santé publique et de marketing alimentaire sont discutées.

\section{La perception du risque alimentaire, corollaire de la diététique}

Un lien consubstantiel entre alimentation et santé, générateur de risque perçu L'alimentation a longtemps été au cœur de la médecine dans l'histoire. En Europe, de l'antiquité au XVIIème siècle, la médecine repose sur la théorie des humeurs d'Hippocrate revisitée par Galien (Ferrières 2006). Dans ce paradigme, la santé dépend certes de la constitution des individus mais aussi de facteurs exogènes comme les saisons, le mode de vie, la position sociale ou la qualité de ce qui est ingéré. Pour éviter de tomber malade et se soigner, il faut adapter son alimentation en équilibrant les quatre types d'humeur - sanguine, colérique, mélancolique ou flegmatique -, par la médecine dite des contraires (Ferrières 2006). Par exemple, pour lutter contre l'excès de bile noire ou mélancolie, on recommande au Moyen-Age de consommer des aliments blancs tels que le lait, le pain à base de farine de froment ou la viande blanche. Philosophiquement, la diététique européenne médiévale se rapproche de celle pratiquée en Chine depuis des millénaires. L'alimentation constitue en effet l'un des cinq piliers de la médecine chinoise antique et doit assurer une harmonie entre le yin et le yang, selon le principe de cinq éléments reliés à cinq organes principaux, humeurs et saisons. Dans le système galénique comme dans la médecine traditionnelle chinoise, une alimentation appropriée a des vertus à la fois préventives et curatives.

Si l'on attribue à l'alimentation le pouvoir de soigner et de maintenir en bonne santé, en corollaire elle peut rendre malade quand les principes diététiques ne sont pas respectés. Elle génère donc aussi de profondes inquiétudes. D'ailleurs, les peurs alimentaires étaient très développées au Moyen-Âge (Ferrières 2006). Contrairement à un préjugé courant, elles n'étaient pas seulement liées aux possibles pénuries, mais déjà à la qualité des aliments et à la peur de s'empoisonner (Pichon, 2012). Dès le Moyen-Age s'est donc mise en place une 
réglementation pour protéger le consommateur. Au XIVème siècle, la Charte de Mirepoix définit les conditions de vente de viande pour assurer une certaine sécurité alimentaire. A la même époque, le codex alimentarius spécifie que le bétail doit entrer sur pied en ville, où sont transférés les abattoirs, afin que chacun puisse s'assurer que les bêtes sont - littéralement bien portantes, c'est-à-dire propres à la consommation (Ferrières 2006). Les autorités ont donc identifié depuis longtemps la nécessité de prendre en compte le risque alimentaire perçu et de rassurer la population par des mesures sanitaires.

\section{La complexité du risque alimentaire}

Toutefois, le risque est un concept complexe à appréhender car il mêle l'objectif et le subjectif. Sa première définition est donnée par Bernoulli en 1738 dans la lignée des travaux sur le jeu de Pascal et Huygens, comme l' «espérance mathématique d'une fonction de probabilités d'événement » (Jousse 2004). Pour autant, la perception d'un même risque varie fortement d'un individu à l'autre selon les caractéristiques psychologiques et le contexte culturel et social. En effet, elle correspond à la vulnérabilité éprouvée par les individus face à l'occurrence et à la gravité d'une menace. Cela explique le décalage fréquent entre le discours des experts et le ressenti du public face à un même risque (Kasperson et al. 1988). Pour analyser les comportements des consommateurs, il apparaît pertinent d'étudier le risque perçu plutôt que le risque objectif, comme le soulignait déjà Bauer (1960), le premier chercheur à avoir promu ce concept en marketing. C'est d'autant plus approprié pour l'alimentation que les aspects psychologiques, sociaux et culturels sont très présents dans les discours des consommateurs français (Laporte, Michel, et Rieunier 2015). Parmi les multiples dimensions $\mathrm{du}$ risque alimentaire (notamment physique, psychologique, sociale, de performance ou financière), nous nous intéressons ici à celle qui est directement liée à la santé de l'individu, la dimension physique du risque alimentaire (Gallen et Cases 2007).

\section{Une évolution dans la perception du risque alimentaire ?}

\section{La distinction conceptuelle entre les risques sanitaire et nutritionnel perçus}

La perception du risque alimentaire est justement au cœur d'un paradoxe relevé par de nombreux chercheurs : manger n'a sans doute jamais été aussi sûr en France, pourtant les consommateurs perçoivent un risque alimentaire élevé pour leur santé (Bergadaà et Urien 2006; Pichon 2012). La typologie réalisée par Ferreira (2006) éclaire ce paradoxe, en 
répartissant les risques alimentaires perçus selon deux axes (Figure 1). L'axe vertical oppose le risque sanitaire au risque nutritionnel en répondant à la question suivante : le risque provient-il d'une contamination par des éléments extrinsèques à l'aliment, ou d'effets secondaires induits par l'alimentation ? L'axe horizontal caractérise la matérialité du risque : est-il physique ou symbolique ? Cette typologie nous permet de catégoriser différents risques alimentaires perçus, ajoutés à titre d'exemples sur la Figure 1. Ainsi, le risque sanitaire correspond à une intoxication consécutive à l'ingestion d'un aliment contaminé par des éléments extrinsèques, comme les bactéries (cadran supérieur gauche de la Figure 1). Mais cette contamination peut aussi être symbolique (cadran supérieur droit). Par exemple, la crise provoquée par la viande de cheval retrouvée en 2013 dans des lasagnes et autres plats préparés n'a a priori causé aucune victime. Toutefois, la viande de cheval a symboliquement contaminé les aliments, car elle ne figurait pas dans les ingrédients déclarés. Elle a en cela remis en cause la confiance des consommateurs mais aussi la traçabilité des produits.

Le risque nutritionnel est plus insidieux puisqu'il concerne les effets secondaires des aliments (Aurier et Sirieix 2009). Le «caractère invisible de l'attribut nutritionnel » rend sa gestion compliquée (Gomez 2008). Ses effets sont diffus, se manifestent à long terme et sont difficiles à isoler et à vérifier, ce qui explique que la nutrition repose sur des attributs de croyance plutôt qu'expérientiels (Larceneux 2003). Le risque nutritionnel perçu est intrinsèquement lié au produit, par exemple à sa teneur en nutriments, mais aussi à la présence de certains produits prévus dans le cahier des charges de l'aliment. Ainsi, la présence de colorants et d'arômes dans la formulation de produits industriels est étudiée pour apporter une apparence et une saveur appétissantes pour les consommateurs, souvent à l'issue de nombreux tests. Elle est culturellement induite selon Ferreira (2006), tout en suscitant chez certains de l'inquiétude quant aux effets à long terme sur la santé. Elle entre donc dans le cadran inférieur droit de la Figure 1. En revanche, les graisses hydrogénées consistant à solidifier des graisses végétales sont utilisées à des fins purement techniques, pour faciliter la production industrielle (par exemple de viennoiseries), et non en réponse à des attentes de consommateurs. Elles génèrent des acides gras trans, accusés de contribuer à long terme à l'obésité, aux maladies cardiovasculaires et au diabète de type deux. Elles concernent donc le cadran inférieur gauche de la Figure 1.

Figure 1 : Distinction entre le risque sanitaire et le risque nutritionnel perçus (adaptée de Ferreira 2006) 
Risque sanitaire perçu

(contamination due à des éléments extrinsèques à l'aliment)

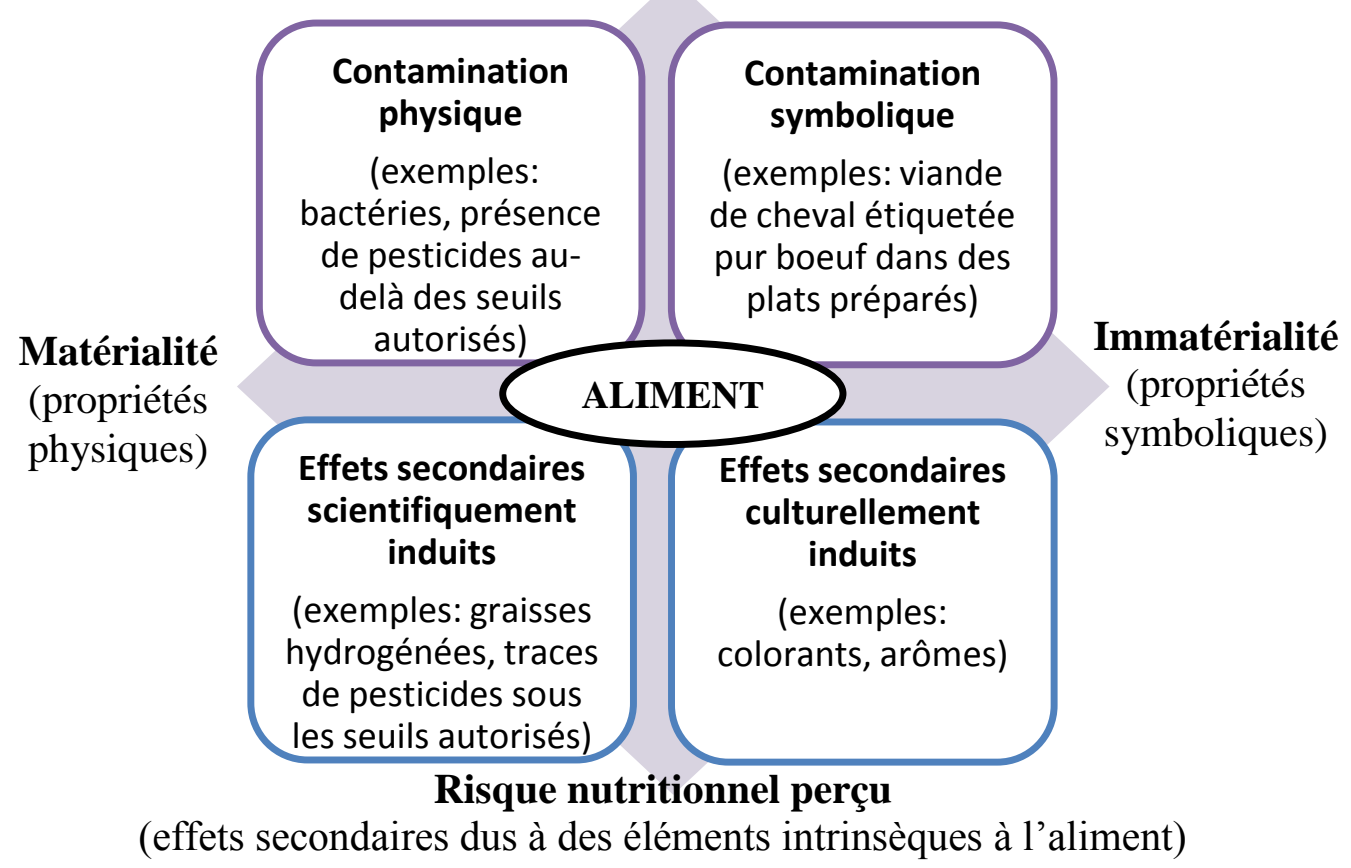

Une frontière ténue entre risque sanitaire et risque nutritionnel perçus

Toutefois, la frontière entre risque sanitaire et risque nutritionnel perçus peut facilement être franchie. D'après Ferreira (2006), tous deux sont conceptuellement corrélés dans l'esprit des consommateurs puisque la présence de l'un entraîne des soupçons sur la présence de l'autre. Le cas des pesticides permet d'illustrer ce continuum entre risque sanitaire et risque nutritionnel perçus. En effet, la législation définit une liste des pesticides autorisés aux différents stades de production dans l'agriculture conventionnelle. Leur présence dans le produit final est tolérée en deçà des seuils réglementaires, les limites maximales de résidus $(\mathrm{LMR})^{2}$. Certes, le consommateur ne connaît pas le niveau des LMR, mais il sait que les produits vendus en Europe doivent respecter la législation. Le respect des limites est spécifié dans le cahier des charges et contrôlé dans les procédures de qualité. La présence de ces pesticides autorisés peut alors être considérée comme intrinsèque à l'aliment, ce qui la place du côté du risque nutritionnel perçu. Par ailleurs, elle est liée au mode de production de certains de ses ingrédients et non pas à une réponse à des attentes culturelles, ce qui la situe du côté des effets secondaires scientifiquement induits (cadran inférieur gauche de la Figure 1).

\footnotetext{
${ }^{2}$ Ou MRL (Maximum Residue Limits). Il s'agit des teneurs maximales de résidus de pesticides ou produits vétérinaires, qui sont déterminées par les pouvoirs publics. Dans l'Union Européenne, elles sont définies par le règlement $n^{\circ} 396 / 2005$.
} 
En revanche, il est interdit de dépasser les LMR pour ces pesticides. De plus, les pesticides non mentionnés sur la liste autorisée sont formellement interdits. Si un aliment s'avérait comporter des résidus de pesticides interdits, ou des pesticides autorisés mais en trop grande quantité, il s'agirait d'une véritable situation de crise, dont le consommateur aurait vraisemblablement connaissance par les médias. En effet, ces pesticides seraient extrinsèques au produit car spécifiquement exclus du cahier des charges, au même titre que des corps étrangers susceptibles d'empoisonner les consommateurs. Ils seraient alors à classer dans le risque sanitaire perçu en tant que contamination physique, dans le cadran supérieur gauche de la Figure 1. Donc si certains des pesticides actuellement autorisés venaient à être retirés du marché, on basculerait d'un risque nutritionnel perçu à un risque sanitaire perçu.

\section{Une prise de conscience du risque nutritionnel récente en France}

Selon le $\mathrm{Crédoc}^{3}$, la représentation sociale d'une «alimentation plaisir » domine en France. Mais elle a reculé à la fin des années 1980 au profit de l' «alimentation santé », parallèlement à l'orientation nutritionnelle des discours publics et notamment au lancement du premier Plan national nutrition santé (PNNS) en 2001. Cette tendance ralentit toutefois depuis 2013, indiquant que les Français cherchent à concilier plaisir et santé. D'un point de vue historique, l'alimentation est en effet sociale et communielle dans la France de tradition catholique influencée par l'approche de Galien, alors qu'elle est individualisée et médicalisée aux EtatsUnis dans la lignée d'un protestantisme renforcé par l'héritage de Paracelse ${ }^{4}$ (Ferrières 2006). Madeleine Ferrières appelle cela la «loi des $2 \mathrm{~S}$ : Sécurité-Santé dans les pays anglo-saxons, Saveur-Sociabilité ailleurs ». D'après les travaux de l'historienne, le contraste entre ces deux visions de l'alimentation s'est installé durablement à la fin du XIXème siècle, avec d'un côté l'avènement des gastronomes en France, de l'autre la New Nutrition aux Etats-Unis dressant la liste des aliments bons pour la santé ou au contraire à proscrire. Cette vision américaine utilitariste et morale de l'alimentation se retrouve dans le lancement concomitant de cornflakes prêts à consommer par John Kellogg. Pour ce prédicateur adventiste et médecin, il faut proposer une alimentation à la fois saine et rapide à préparer et consommer, car manger ne serait constituer « un passe-temps, un divertissement [ni] un plaisir ». Il se situe donc à l'opposé de l'«hédonisme alimentaire qui met la santé entre parenthèses » à cette même époque en France (Ferrières 2006).

\footnotetext{
${ }^{3}$ Les enquêtes Comportements et consommation alimentaire en France (CCAF) sont menées par le Crédoc tous les 3 ans depuis 1999.

${ }^{4}$ Ce médecin a dénoncé au début du XVI siècle la médecine des humeurs de Galien et prôné une approche expérimentale combattant les causes externes de la maladie grâce à la chimie.
} 
Mais un siècle plus tard, le contexte culturel et social a profondément évolué en France. A partir des années 1980, le marketing agro-alimentaire, les politiques publiques, les médias portent un discours orienté vers la nutrition. De nouveaux produits apparaissent, les alicaments, contraction des mots aliments et médicaments, dont l'emblématique Bio (Activia) de Danone, lait fermenté au bifidus actif lancé en 1987. La part de produits transformés dans l'alimentation des Français augmente fortement, parallèlement au travail généralisé des femmes et à l'urbanisation croissante ; elle représente en 2017 plus de la moitié des aliments consommés par les adultes hors établissements de restauration, plus des deux-tiers chez les enfants ${ }^{5}$. Enfin, une série de scandales alimentaires affecte la confiance des consommateurs à partir de la fin des années quatre-vingt-dix. Ces crises sont le plus souvent sanitaires puisqu'elles sont dues à une contamination physique ou symbolique de l'aliment par un élément extrinsèque. Elles ont pour conséquence un renforcement des contrôles et des normes. En particulier, la crise de la vache folle a marqué les esprits et conduit en 2000 à la création de l'Agence française de sécurité sanitaire des aliments (Afssa). Le risque sanitaire, autrefois vu comme une fatalité, est désormais considéré comme exceptionnel, mais d'autant plus intolérable qu'il peut être évité.

Ironiquement, les mesures prises contre le risque sanitaire pourraient accroître la perception de risque nutritionnel. En effet, la standardisation des procédés industriels introduit une distance entre l'alimentation et les représentations que s'en fait le consommateur, ce qui provoque une dissonance cognitive, donc une perception de risque et un besoin de réassurance (Gallen 2001). D'après Fischler (2001), «la peur est dans l'assiette » pour les Français désormais en perte de repères. Plus que des produits avariés (risque sanitaire), ils semblent s'inquiéter des « toxicités en partie bien réelles de la modernité, les additifs, les colorants, les pesticides » (risque nutritionnel). Certes, la réglementation protège les consommateurs en spécifiant la liste des additifs et pesticides autorisés, en imposant des LMR et en procédant à des contrôles. Mais les consommateurs redoutent les effets secondaires de ces produits à long terme sur leur santé et un éventuel effet cocktail. Or, les recherches montrent que des risques non observables, difficilement contrôlables, inconnus de ceux qui y sont exposés et aux effets éloignés dans le temps amplifient leur perception auprès du public, a fortiori quand les conséquences peuvent affecter la santé, voire être fatales (Slovic 1987). Jusqu'à la fin du

\footnotetext{
${ }^{5}$ Etude individuelle nationale des consommations alimentaires 3 (INCA3) de l'Anses (Agence nationale de sécurité sanitaire de l'alimentation, de l'environnement et du travail) parue en 2017.
} 
XIXème siècle, les consommateurs en lutte contre la pénurie étaient dans une logique de quantité plutôt que de qualité (Pichon 2012). Proches d'une alimentation souvent produite par leurs soins ou par des personnes de leur connaissance, ils s'inquiétaient d'autant moins des effets à long terme sur leur santé que leur espérance de vie était moitié moindre qu'aujourd'hui : «on sera morts avant, sans doute d'autre chose », rétorquaient les patients aux médecins qui tentaient de les sensibiliser à la nutrition (Ferrières 2006).

L'évolution du contexte culturel, économique et social laisse envisager une évolution du risque alimentaire perçu. Afin de mesurer, d'une part, si les consommateurs distinguent bien les deux dimensions théoriques du risque alimentaire, d'autre part, si la perception du risque nutritionnelle est aujourd'hui prépondérante, deux études quantitatives sont réalisées auprès de consommateurs français.

\section{La perception du risque alimentaire chez les consommateurs français}

\section{La méthodologie des deux études quantitatives}

Deux collectes de données en ligne sont effectuées auprès de 280 et 783 personnes, âgées en moyenne de 42 ans et réparties dans toute la France. La première utilise le panel d'une société d'études de façon à représenter la répartition géographique et socio-économique de la population française $e^{6}$. La seconde repose sur un échantillon de convenance constitué par boule de neige à partir du réseau de l'auteur. Cette deuxième collecte permet de fortement augmenter la taille de l'échantillon et donc de distinguer les résultats par profils. Mais elle perd en représentativité géographique ${ }^{7}$ et socio-professionnelle ${ }^{8}$ en surreprésentant la région parisienne et les catégories les plus élevées. La répartition par âge est sensiblement identique dans les deux collectes ${ }^{9}$.

Les répondants sont projetés dans la situation suivante. Un soir de semaine à la maison, n'ayant plus grand-chose à manger chez eux, ils se rendent dans un magasin pour acheter de quoi rapidement préparer un dîner : un plat principal et un dessert. Les aliments diffèrent entre

\footnotetext{
${ }^{6}$ L'auteur remercie son laboratoire pour le financement de cette collecte auprès de la société Créatest.

${ }^{7}$ Par région identifiée selon les indicatifs téléphoniques : 01-Ile de France 70\%, 02-Nord-Ouest 12\%, 03-NordEst 5\%, 04-Sud-Est 9\%, 05-Sud-Ouest 4\%.

${ }^{8} 41 \%$ des répondants dans les catégories supérieures, 30\% dans les intermédiaires, $29 \%$ dans les moins élevées

${ }^{9}$ Respectivement $36 \%$ et $38 \%$ de moins de 35 ans, $33 \%$ et $35 \%$ entre 35 et 49 ans, $29 \%$ de plus de 50 ans
} 
les deux collectes afin d'augmenter la validité externe des résultats, mais les questionnaires sont par ailleurs semblables (Encadré 1). Les produits suivants sont présentés aux répondants :

- Etude 1 : risotto aux légumes (vendu à température ambiante dans le rayon épicerie) et mousse au chocolat (frais libre-service).

- Etude 2 : demi-lunes tomate mozzarella (pâtes fraîches fourrées, vendues au rayon frais libre-service), et crème caramel (frais libre-service) ou pomme golden (frais traditionnel, en vrac dans le rayon fruits et légumes sur la photo soumise aux répondants).

Ces produits sont sélectionnés de façon à représenter les trois principaux rayons d'achat en grande distribution ${ }^{10}$ et à faire varier les modes de conservation (à température ambiante ou au frais), le degré de transformation des aliments ${ }^{11}$ et le type de contamination ou d'effets secondaires envisageables selon la Figure 1. Ainsi, la présence d'œufs et de produits laitiers dans les pâtes et la crème caramel pourrait accroître le risque sanitaire perçu par contamination physique. De même, les pommes, fruit le plus consommé en France, donnent régulièrement lieu à des articles sur la présence de pesticides ${ }^{12}$. De plus, contrairement aux autres produits testés, elles sont vendues en vrac dans l'expérimentation, et peuvent donc être perçues comme contaminées par d'autres consommateurs qui les auraient manipulées (Argo, Dahl, et Morales 2006). Enfin, afin d'éviter que le prix et la marque ne biaisent le risque perçu, les produits retenus sont tous positionnés en milieu de gamme, dans un packaging qui a permis d'effacer toute référence à la marque avec un logiciel de traitement de l'image.

\section{Encadré 1 : Les principales variables mesurées}

Après avoir contrôlé que les personnes ont déjà mangé le type d'aliments présenté et ne manifestent pas d'aversion à leur encontre, on mesure les perceptions de risque sanitaire et de risque nutritionnel en adaptant l'échelle de Cunningham (1967). Cette échelle, largement employée en marketing (Larceneux 2003; Volle 1995), multiplie entre elles les deux dimensions du risque perçu: (1) sa probabilité d'occurrence et (2) la gravité de ses conséquences. Ces deux items sont évalués sur une échelle de Likert en 7 points («pas du tout d'accord » à « tout à fait d'accord »). Leur produit mesure le risque perçu en 49 points.

\footnotetext{
${ }^{10}$ Le frais libre-service représente $22 \%$ du chiffre d'affaires, l'épicerie $18 \%$ et le frais traditionnel $17 \%$ selon l'étude Nielsen et Bonial de 2016.

${ }^{11}$ Rappelons que plus de la moitié de l'alimentation des adultes français se compose de produits transformés (étude INCA3 précédemment citée).

${ }^{12}$ Suite notamment aux rapports de la European food safety agency (Efsa) et aux études menées par des Organisations Non Gouvernementales.
} 
- Perception du risque nutritionnel (PRN) : si quelqu'un mange régulièrement cet aliment, (1) cela aura sûrement des effets à long terme sur son état général (santé, apparence, bienêtre...) ; (2) ces effets à long terme sur son état général seront nocifs.

- Perception du risque sanitaire (PRS) : si quelqu'un mange cet aliment ce soir, (1) il risque fort de tomber malade dans les jours suivants ; (2) cette maladie sera dangereuse pour la santé de la personne.

L'intention d'achat est ensuite mesurée sur une échelle de Likert en 7 points. Enfin, des éléments sociodémographiques sont récoltés.

\section{Les résultats : une perception de risque nutritionnelle dominante}

Un test de comparaison de moyennes montre que pour chacun des aliments étudiés, les consommateurs perçoivent significativement plus de risque nutritionnel que de risque sanitaire (Tableau 1). La crème caramel et les pâtes à la mozzarella sont les produits générant le plus de risque perçu, tant sanitaire que nutritionnel. Ce sont les deux seuls comportant à la fois des œufs et des produits laitiers parmi leurs ingrédients, pourtant le risque perçu est essentiellement nutritionnel. Les trois autres produits étudiés suscitent des perceptions du risque très faibles sur le plan sanitaire, un peu plus élevées sur le plan nutritionnel.

Tableau 1 : Comparaison des perceptions de risque sanitaire (PRS) et nutritionnel (PRN)

\begin{tabular}{lccccccc}
\hline & \multicolumn{2}{c}{$\begin{array}{c}\text { PRS } \\
(/ 49)\end{array}$} & \multicolumn{2}{c}{$\begin{array}{c}\text { PRN } \\
(/ 49)\end{array}$} & \multicolumn{3}{c}{$\begin{array}{r}\text { Test de comparaison de moyennes } \\
\text { entre PRS et PRN }\end{array}$} \\
\cline { 2 - 7 } & Moyenne & $\begin{array}{c}\text { Ecart- } \\
\text { type }\end{array}$ & Moyenne & $\begin{array}{c}\text { Ecart- } \\
\text { type }\end{array}$ & $\begin{array}{c}\text { Degré de } \\
\text { liberté }\end{array}$ & Test t & $\mathrm{p}$ \\
\hline $\begin{array}{l}\text { Risotto } \\
\text { légumes }\end{array}$ & 3,30 & 3,41 & 9,92 & 7,53 & 279 & $-15,58$ & $0,000^{* * *}$ \\
$\begin{array}{l}\text { Mousse } \\
\text { chocolat }\end{array}$ & 3,94 & 4,37 & 11,23 & 10,16 & 279 & $-12,86$ & $0,000^{* * *}$ \\
$\begin{array}{l}\text { Pâtes } \\
\text { mozzarella }\end{array}$ & 4,23 & 4,71 & 28,96 & 13,53 & 782 & $-51,04$ & $0,000^{* * *}$ \\
$\begin{array}{l}\text { Crème } \\
\text { caramel }\end{array}$ & 5,40 & 6,72 & 30,14 & 14,26 & 751 & $-46,52$ & $0,000^{* * * *}$ \\
$\begin{array}{l}\text { Pommes } \\
\text { golden }\end{array}$ & 3,40 & 4,46 & 11,82 & 9,97 & 743 & $-25,63$ & $0,000^{* * *}$ \\
\hline
\end{tabular}

Significativités de p aux seuils de 0,001\%***, 0,01\%**,0,05\%*,0,10\% , NS non significatif

Tableau 2 : Coefficients de corrélation entre PRS et PRN 


\begin{tabular}{lccccc}
\hline \multicolumn{1}{c}{ Produit } & $\begin{array}{c}\mathrm{N} \\
\text { (effectif) }\end{array}$ & $\begin{array}{c}\mathrm{r} \text { (coefficient de } \\
\text { corrélation de } \\
\text { Pearson) }\end{array}$ & $\mathrm{p}$ & \multicolumn{2}{c}{$\begin{array}{c}\text { Intervalle de confiance } \\
\text { calculé par boostrap }\end{array}$} \\
\hline $\begin{array}{l}\text { Risotto } \\
\text { légumes }\end{array}$ & 280 & 0,35 & $0,000^{* * * *}$ & 0,23 & Inférieur \\
$\begin{array}{l}\text { Mousse } \\
\text { chocolat }\end{array}$ & 280 & 0,37 & $0,000^{* * *}$ & 0,22 & 0,56 \\
$\begin{array}{l}\text { Pâtes } \\
\text { mozzarella }\end{array}$ & 783 & 0,17 & $0,000^{* * *}$ & 0,10 & 0,23 \\
$\begin{array}{l}\text { Crème } \\
\text { caramel }\end{array}$ & 752 & 0,19 & $0,000^{* * *}$ & 0,12 & 0,24 \\
$\begin{array}{l}\text { Pommes } \\
\text { golden }\end{array}$ & 744 & 0,44 & $0,000^{* * *}$ & 0,34 & 0,53 \\
\hline
\end{tabular}

Significativités de p aux seuils de 0,001\%***, 0,01\%**, 0,05\%*, 0,10\% ${ }^{\circ}$ NS non significatif

Les coefficients de corrélation de Pearson calculés pour les cinq produits montrent que la PRS et la PRN sont positivement corrélées pour les consommateurs : quand l'une augmente, l'autre aussi (Tableau 2). C'est surtout le cas pour les pommes, sans doute à cause des éventuels résidus de pesticides, qui peuvent susciter une perception de risque nutritionnel audessous des limites autorisées, sanitaire au-dessus. Dans les cinq cas, la PRN est bien distincte de la PRS. En effet, tous les coefficients sont éloignés de la valeur 1, qui est exclue des intervalles de confiance calculés par bootstrap. Les répondants ont donc bien distingué les deux dimensions du risque alimentaire.

Des régressions linéaires montrent que plus les individus perçoivent de risque nutritionnel pour un produit, moins ils ont l'intention de l'acheter $\left(\beta_{\text {Risotto }}=-0,28 ; \beta_{\text {Mousse }}=-0,21 ; \beta_{\text {Pâtes }}=\right.$ $\left.-0,19 ; \beta_{\text {Crème }}=-0,29 ; \beta_{\text {Pommes }}=-0,38 ; p \leq 0,001 * * *\right)$. La PRN a donc une influence négative sur l'intention d'achat. De plus, prendre en compte la PRN conjointement à la PRS (après avoir vérifié l'absence de colinéarité) permet d'améliorer significativement la prédiction d'intention d'achat (de 2 à 8 points, $p=0,000^{* * *}$ ). Notons toutefois que la PRS et la PRN n'expliquent ensemble qu'une part limitée de l'intention d'achat $\left(R^{2}{ }_{\text {Risotto }}=13 \% ; R^{2}\right.$ Mousse $=$ $19 \% ; R_{\text {Pâtes }}^{2}=8 \% ; R_{\text {Crème }}^{2}=9 \% ; R^{2}$ Pommes $\left.=16 \%\right)$.

Tableau 3 : Différences de PRS et PRN selon les profils de répondants dans l'étude 2

${ }^{13}$ La procédure boostrap génère des échantillons répétés et estime un intervalle de confiance fiable en s'affranchissant des conditions de normalité et d'homogénéité des variances. Ici, les intervalles de confiance à 95\% sont calculés par échantillons répétés fixés à 2000 par la technique de Bias Corrected and accelerated. 


\begin{tabular}{llcc}
\hline & Produit & Différence de PRN & Différence de PRS \\
\hline Femmes vs Hommes & Pâtes & $+3,23 ; \mathrm{p}=0,006^{* *}$ & NS \\
& Crème & $+2,28 ; \mathrm{p}=0,066^{\circ}$ & NS \\
\multirow{2}{*}{ Jeunes adultes vs Seniors } & Pâtes & $+4,74 ; \mathrm{p}=0,010^{* *}$ & $-3,42 ; \mathrm{p}=0,010^{* *}$ \\
& Crème & $+7,89 ; \mathrm{p}=0,000^{* * *}$ & $-2,14 ; \mathrm{p}=0,046^{*}$ \\
& Pommes & $-3,34 ; \mathrm{p}=0,020^{* *}$ & $-1,45 ; \mathrm{p}=0,037^{*}$ \\
\multirow{2}{*}{ Ruraux vs Urbains } & Pommes & $+3,84 ; \mathrm{p}=0,006^{* *}$ & $+2,21 ; \mathrm{p}=0,005^{* *}$ \\
Nord-Ouest vs Ile de France & Pommes & $+6,29 ; \mathrm{p}=0,000^{* * *}$ & $+2,16 ; \mathrm{p}=0,000^{* * *}$ \\
\hline Significativités de $p$ aux seeils & &
\end{tabular}

Significativités de p aux seuils de 0,001\%***, 0,01\%**, 0,05\%*, 0,10\% , NS non significatif

La taille importante de l'échantillon dans l'étude 2 permet d'affiner les résultats selon le profil des répondants (Tableau 4). La PRN des femmes envers les produits transformés apparaît légèrement supérieure à celle des hommes, alors que les PRS sont similaires. L'âge joue un rôle, la plus grande différence se manifestant entre les deux générations les plus éloignées, de manière inversée entre la PRN et la PRS. Ce sont en effet les jeunes adultes qui manifestent la plus grande PRN, et les seniors, la plus grande PRS. Enfin, le lieu d'habitation influence la PRN et la PRS envers les pommes (dont on a vu qu'elles sont assez fortement corrélées) : les personnes habitant dans des milieux ruraux, notamment dans le Nord-Ouest, importante zone de production de pommes, perçoivent plus de risque que les urbains et en particulier les Parisiens, sur les plans tant sanitaire que nutritionnel.

\section{Les implications de la perception du risque alimentaire}

\section{La pertinence de distinguer risque sanitaire et nutritionnel perçus}

Cette recherche vérifie empiriquement l'intérêt de distinguer les deux dimensions théoriques du risque alimentaire perçu. En effet, les répondants établissent une différence entre la perception du risque sanitaire et la perception du risque nutritionnel. De plus, les résultats montrent que la seconde a pris le pas sur la première chez les consommateurs français interrogés : aujourd'hui, ils s'inquiètent davantage des effets secondaires à long terme de leur alimentation que des risques d'intoxication à court terme, comme le supposait la littérature. Celle-ci permet d'éclairer l'effet générationnel constaté, les jeunes adultes percevant plus de risque nutritionnel que les seniors mais moins de risque sanitaire : en France, par héritage historique, la prise de conscience de la dimension nutritionnelle est récente et le risque alimentaire était jusqu'à récemment principalement réduit à sa dimension sanitaire. Enfin, les 
résultats montrent que la perception de l'une nourrit des soupçons sur la présence de l'autre, comme le pressentait sur un plan conceptuel Ferreira (2006). En effet, les perceptions de risque nutritionnel et de risque sanitaire sont corrélées d'après les analyses statistiques effectuées, surtout pour les pommes, produit brut fortement traité. Or, on a vu que la présence de pesticides fait basculer la perception de risque du côté sanitaire si les résidus sont audessus des LMR. Cela peut expliquer pourquoi les personnes habitant en milieu rural, conscientes des modes de production et en particulier de la quantité de pesticides utilisée, perçoivent plus de risque que les urbains envers les pommes.

Il apparaît donc essentiel que les pouvoirs publics prennent en compte la perception du risque nutritionnel afin d'encourager des comportements alimentaires plus sains, dans un contexte où la part des aliments transformés s'accroît. Les deux études quantitatives ont montré que la perception de risque nutritionnel envers un aliment diminue l'intention d'achat de ce produit. Il faudrait donc alerter les consommateurs quand un produit présente un risque nutritionnel avéré, ou les rassurer dans le cas contraire. Il est aussi dans l'intérêt des entreprises de prendre en considération la perception du risque nutritionnel des consommateurs puisque cela influe sur les ventes. C'est donc un levier pour inciter les entreprises à reformuler leurs produits afin de les rendre plus sains.

Mais en même temps et de façon plus contre-intuitive, cette recherche apporte des arguments à l'Anses ${ }^{5}$ qui s'inquiète d'une recrudescence des prises de risque alimentaire sanitaire, tout particulièrement chez les jeunes adultes urbains. L'agence pointe une augmentation de «la consommation de denrées d'origine animale (œuf, viande, poisson, mollusque) crues (...), notamment sous forme de sushis » et le non-respect des dates limites de conservation pour des produits à risque (car moins transformés) tels que les préparations culinaires maison ou issues de circuits courts. La présente recherche permet de l'expliquer. Elle montre que les Français se préoccupent aujourd'hui davantage du risque nutritionnel que leurs aînés, mais peuvent aussi sous-estimer le risque sanitaire. Habitués depuis plusieurs générations à consommer des produits industrialisés très contrôlés sur le plan sanitaire, ils tiennent pour acquise l'absence de contamination. Sauf que de plus en plus de consommateurs privilégient aujourd'hui les aliments moins transformés et sans conservateurs. Or, ceux-ci requièrent des conditions de conservation plus strictes et sont loin d'être exempts de risque, comme le rappelle l'importante crise sanitaire européenne provoquée en 2011 par la contamination bactérienne de graines germées issues de l'agriculture biologique à l'excellente image nutritionnelle. 
L'Anses relève d'ailleurs une «augmentation de toxi-infections alimentaires collectives survenues dans le cadre familial ». Il apparaît donc nécessaire de relancer des campagnes d'éducation à des règles d'hygiène que l'on croyait à tort acquises, plus particulièrement auprès des jeunes générations urbaines. Il se dessine donc l'importance de communiquer sur les deux types de risque alimentaire mais dans une approche différenciée selon les publics.

Il convient toutefois de noter que la PRS et la PRN n'expliquent ensemble qu'une partie limitée de l'intention d'achat d'après les études réalisées. Cela s'explique sans doute par l'importance de facteurs autres que la «sécurité et la santé » dans les choix alimentaires des Français, comme la «saveur et la sociabilité » (Ferrières 2006), encore très présentes dans le modèle culturel d'après les études du Crédoc précédemment mentionnées. Cela confirme l'intérêt pour les pouvoir publics de ne pas communiquer uniquement sur les aspects sanitaires et nutritionnels de l'alimentation, mais de prendre aussi en compte le plaisir et la commensalité, comme le fait le Programme National Alimentation (PNA), alors que le Programme National Nutrition Santé (PNNS) se focalise sur les aspects nutritionnels.

\section{Les limites des solutions classiques pour « marketer » la nutrition}

Si les informations sur la sécurité alimentaire paraissent simples à diffuser car crédibles et facilement vérifiables, il en est tout autre pour le risque nutritionnel. En effet, les consommateurs accordent une confiance limitée envers le discours nutritionnel des marques et en sont de ce fait souvent réduits à se faire leur propre idée (Guichard et Muratore 2011). Par exemple, des marques de céréales pour petit-déjeuner mettent en avant des bénéfices nutritionnels dans leurs publicités et en face avant de leurs emballages, comme la présence de fibres, de calcium, de fer et de plusieurs vitamines. Pour autant, cela ne garantit pas un profil nutritionnel équilibré au global. Les produits peuvent comporter par ailleurs une grande quantité de sucre ou de sel, discrètement mentionnée dans les tableaux nutritionnels à l'arrière du paquet. C'est ce que dénonce le bureau européen des unions de consommateurs (BEUC), appelant à un changement de réglementation.

Pour signaler la qualité nutritionnelle des produits, il apparaît dès lors plus efficace de faire appel à un tiers de confiance, dépourvu d'intérêt commercial, plutôt qu'à l'industriel ou au distributeur qui peuvent être soupçonnés de partialité. C'est le cas avec les labels (Larceneux 2003) et l'étiquetage nutritionnel (Nabec 2017), reposant sur l'émetteur du label ou le 
législateur. Toutefois, les effets de ces signaux sont limités car leur prise en compte dépend des capacités cognitives des consommateurs, de leurs motivations individuelles et d'heuristiques de lecture qui parfois introduisent des biais décisionnels (Nabec 2017). En effet, compte-tenu de la complexité de l'information, les consommateurs ne l'utilisent pas en intégralité mais ils sélectionnent les éléments qui répondent à leurs objectifs, qu'ils soient négatifs (par exemple réduire le sucre) ou positifs (par exemple augmenter la quantité de calcium). Cela simplifie certes le processus de décision, mais cela néglige des aspects clés. Cela peut résulter en une évaluation erronée du profil nutritionnel par effet de halo, en surestimant les éléments négatifs de l'aliment (biais de négativité) ou au contraire en les sousestimant (biais de positivité). Ce processus a été documenté dans de nombreuses recherches (voir Nabec 2017 pour une synthèse).

De plus, pour être reconnus, les signaux nécessitent d'être largement adoptés par l'industrie. Cela peut expliquer les résultats en demi-teinte d'un label récemment développé par les pouvoirs publics français, le Nutri-Score. L'objectif du Nutri-Score, dont l'usage est facultatif, est de rendre l'information nutritionnelle plus accessible. Apposé en face avant des emballages, ce logo comporte cinq couleurs, du vert foncé au rouge, et cinq lettres de A à E. Il note ainsi le profil nutritionnel du produit dans son ensemble. A caractère interprétatif, il est mieux compris par les consommateurs que les logos nutritionnels descriptifs (Mérigot et Nabec 2016). Mais son utilisation repose sur le volontariat des entreprises. Or, nombre d'entre elles refusent à ce jour de l'utiliser. Elles le justifient par la crainte de diaboliser certains aliments et d'encourager des comportements d'orthorexie alimentaire en allant à l'encontre du plaisir de manger. Elles soulignent que l'équilibre alimentaire ne dépend pas seulement de la qualité des aliments ingérés, mais aussi de l'hygiène de vie, des quantités ingérées et des fréquences des prises, puisque l'équilibre se construit sur plusieurs repas (Mérigot et Nabec 2016). L'ensemble des freins relevés dans le recours aux solutions classiques est synthétisé dans le Tableau 4.

\section{Les applications digitales nutritionnelles, un outil de réassurance ?}

Une solution semble porteuse, le recours à des applications mobiles nutritionnelles qui décodent les étiquettes, telles que Yuka (Encadré 2). Leur utilisation s'est multipliée au cours des dernières années et les trois quarts des utilisateurs affirment qu'elles ont modifié leur 
comportement d'achat ${ }^{14}$. Ces applications apportent des réponses à la perception du risque nutritionnel, récapitulées dans le Tableau 4, en dépassant de nombreux freins parmi ceux précédemment cités. Certes, elles nécessitent toujours une démarche active de la part du consommateur, puisqu'il lui appartient de charger l'application puis de l'utiliser ou non. Mais le format interactif favorise l'appropriation par les consommateurs (Gallen, Pantin-Sohier, et Peyrat-Guillard 2019). Ces applications permettent d'accéder à la composition détaillée des produits, sans souci de taille des caractères, ce qui la rend plus lisible que sur les emballages. De plus, le caractère ludique du format et la présence d'une note évaluant le profil nutritionnel global du produit rendent ces informations plus simples à comprendre. Les utilisateurs peuvent par ailleurs personnaliser l'information en intégrant leurs motivations individuelles dans leur profil. L'accès à l'information sur leur smartphone facilite une utilisation routinière, à même d'induire de nouvelles heuristiques (Gallen 2001; 2005). En effet, ces applications analysent les produits scannés mais recommandent aussi des alternatives, créant une architecture de choix incitant à sélectionner le produit qui obtient le meilleur score nutritionnel. De ce fait, Yuka simplifie l'expérience d'achat des consommateurs en allégeant la charge mentale de la décision ${ }^{15}$. Cela correspond à une technique de nudge éprouvée pour ses capacités à changer les comportements (Thaler et Sunstein 2008). Enfin, ces applications autonomisent le consommateur par rapport à l'industriel et au distributeur (consumer empowerment), puisque l'accès à l'information ne repose plus sur ces derniers. Cela modifie le rapport de force et incite les marques à améliorer la composition de leurs produits obtenant de mauvais scores.

Encadré 2 : Un exemple d'application nutritionnelle mobile, Yuka

Yuka est l'application nutritionnelle la plus utilisée en France, avec 8,5 millions d'utilisateurs scannant quotidiennement 2 millions de produits en janvier 2019. Créée en janvier 2017 par une start-up, «l'appli qui déchiffre l'indéchiffrable », comme elle se définit elle-même, affiche pour objectif de «rendre plus transparente la composition des produits » et d'ainsi aider les consommateurs «à s'y retrouver dans la jungle des produits alimentaires pour choisir les bons produits pour sa santé ${ }^{16}$. L'utilisateur scanne le code barre d'un aliment

\footnotetext{
${ }^{14}$ Etude Kantar Worldpanel parue en septembre 2018

15 Etude qualitative réalisée par Unknown en 2019 auprès de 6 hommes et 12 femmes ("Evaluating the Yuka 'phenomenon': How effective is the scanning app in practice?", FoodNavigator.com)

${ }^{16}$ Dossier de presse du 11/02/2019
} 
avec son smartphone et visualise la note sur 100 attribuée au produit ainsi que son code couleur (de vert à rouge). L'application détaille la qualité nutritionnelle calculée selon la méthode du Nutri-Score (60\% de la note), la présence d'additifs $(30 \%)$ et le caractère biologique ou non (10\%). Elle recommande des alternatives de produits obtenant un meilleur score, ce qui a conduit de grandes marques à revoir la composition de leurs produits d'après les dirigeants de la start-up. L'application permet aux utilisateurs de mesurer la qualité nutritionnelle de leur alimentation sur une durée longue grâce à l'enregistrement des données, répondant en cela aux objections soulevées par l'industrie agroalimentaire sur la quantité et la fréquence de consommation. Les informations proviennent d'une large base de données participative en accès ouvert, alimentée et validée par la communauté des utilisateurs. Depuis fin juin 2018, Yuka intègre aussi les cosmétiques et les produits d'hygiène. L'application est saluée par la presse et les consommateurs pour la transparence apportée, mais elle est critiquée pour la diabolisation d'aliments qui présentent pourtant un intérêt nutritionnel mais sont par nature gras, comme le fromage ou l'huile d'olives, ou sucrés, comme le miel. En réponse, la société a développé deux outils : un blog gratuit d'information pédagogique sur l'alimentation, incitant par exemple à consommer du beurre en quantité modérée («10 à $15 \mathrm{~g}$ par jour, soit l'équivalent d'un petit beurre servi en portion individuelle au restaurant ») et un programme nutrition payant incluant un coaching par un nutritionniste.

Tableau 4 : Les applications nutritionnelles mobiles comme outil de réassurance

Freins relevés dans le recours aux solutions classiques

Mise en avant de bénéfices nutritionnels isolés sans tenir compte du profil nutritionnel global du produit

Capacités cognitives limitées des consommateurs

Motivations individuelles variées

Heuristiques de lecture conduisant à des biais décisionnels en se focalisant sur certaines informations au détriment d'autres

\section{Réponses apportées par les applications} nutritionnelles mobiles

Evaluation du profil nutritionnel global du produit et note détaillée par nutriments

Informations nutritionnelles présentées visuellement de façon plus simple, plus lisible et plus attractive que sur un emballage

Possibilité de personnalisation selon les profils individuels

Architecture de choix incitant à sélectionner le produit le plus sain au global (nudge) 
Nécessité d'adoption par les industriels et / ou les distributeurs
Utilisation laissée à l'initiative du consommateur, indépendamment des industriels et des distributeurs

Les applications semblent donc à même de répondre à la perception du risque nutritionnel des consommateurs, les alertant ou les rassurant sur la composition des aliments, incitant les industriels à améliorer celle-ci, et contribuant à l'éducation nutritionnelle des utilisateurs. Elles pourraient même inciter les malades chroniques tels que les diabétiques à une meilleure observance nutritionnelle, apportant ainsi une réponse à un souci de santé publique. Elles permettent donc de «marketer» la nutrition. Pour autant, ces applications présentent d'importantes limites à l'heure actuelle. Elles nécessitent d'utiliser des données fiables, objectives, sérieusement documentées et régulièrement mises à jour. Elles portent aussi une vision manichéenne de l'alimentation, reposant sur la comparaison de profils nutritionnels d'aliments considérés chacun individuellement, sans prendre en compte la taille des portions consommées et l'équilibre global de l'alimentation dans toute sa variété. Enfin, elles diabolisent certains aliments, alors qu'ils ont par ailleurs une utilité nutritionnelle ou sanitaire (comme certains conservateurs par ailleurs naturels et jugés sans risques par les scientifiques). Elles risquent ainsi de développer une approche algorithmique de l'alimentation rejetant arbitrairement certains produits.

Associer les pouvoirs publics à ces outils permettrait de dépasser ces limites, en légitimant la source d'informations et en la replaçant dans le cadre plus large de l'équilibre alimentaire. Ainsi, on a vu que le score de Yuka repose sur trois notes. La première, évaluant les qualités nutritionnelles de l'aliment, repose depuis quelques mois sur le Nutri-Score promu par les pouvoirs publics, dont l'efficacité pour améliorer les comportements alimentaires a été confirmée par des recherches scientifiques. La troisième, portant sur le caractère biologique ou non, peut elle aussi reposer sur des labels externes. Mais la note de 30\% relative aux additifs est contestée par certains experts lui reprochant son caractère arbitraire ${ }^{17}$. Elle pourrait s'appuyer sur des indices scientifiquement validés, qui évaluent le degré de transformation des produits et font l'objet de nombreuses publications, comme la classification NOVA répartissant les aliments en quatre groupes, ou SIGA en huit groupes ${ }^{18}$. Les produits les plus transformés obtiendraient la note la plus faible puisque les études montrent l'intérêt de limiter

\footnotetext{
${ }^{17}$ Consultation Nutrition $\mathrm{n}^{\circ} 86$ 2018, lettre d'information pour professionnels de santé

${ }^{18}$ Information Diététique n ${ }^{\circ} 42017$, revue d'information pour diététiciens
} 
leur consommation. De plus, les quantités indicatives recommandées par les pouvoirs publics seraient précisées, ce qui permettrait de dépasser la vision manichéenne de l'alimentation actuellement reprochée aux applications.

Cette plus grande transparence requise nécessite toutefois des éléments de preuve, car aujourd'hui, $28 \%$ des Français ne font par exemple pas confiance à la liste d'ingrédients ${ }^{14}$. Outre les pouvoirs publics, il serait souhaitable d'associer l'ensemble de la filière, du producteur au distributeur en passant par les industriels, pour garantir la traçabilité et l'authenticité des données. L'utilisation de nouvelles technologies, comme les capteurs connectés et la blockchain réputée incorruptible, parait à ce titre prometteuse. Les applications nutritionnelles qui recueillent des données ainsi garanties et les analysent selon des méthodes scientifiquement validées pourraient alors faire l'objet d'une certification par les pouvoirs publics. Cela leur donnerait les dimensions d'expertise, de labellisation par un tiers indépendant et donc de confiance en une information complète et précise, sachant qu'elles combinent déjà l'expérience et l'interaction. Or, ce sont là autant de facteurs de réassurance pour les consommateurs (Gallen 2001; Gallen et Cases 2007). Les applications digitales nutritionnelles répondraient ainsi aux trois stratégies de réduction de risque alimentaire identifiées par Brunel et Pichon (2004) en dehors de l'évitement : la confrontation, grâce au détail donné sur les produits et aux recommandations alternatives ; la simplification, grâce à la confiance envers la source d'information; la clarification, grâce à la lisibilité et à l'accessibilité d'informations indépendantes. Cette accessibilité pourrait même être élargie audelà des seuls détenteurs de smartphones si les distributeurs proposent ces informations dans leurs propres magasins, via par exemple les bornes de scannage de codes-barres et QR codes. Cela contribuerait en retour à l'image des distributeurs soucieux d'améliorer la santé de l'ensemble de leurs clients.

Pour autant, il reste utopique de réunir toutes les informations nutritionnelles dans un score unique, car les situations individuelles et sociales jouent elles aussi un rôle majeur. Les applications apportent une réponse certes intéressante mais non suffisante. L'amélioration des comportements alimentaires requiert une approche systémique, prenant en compte les contextes d'achat et de consommation, et liant l'ensemble des acteurs. Il apparaît plus que jamais nécessaire d'apporter une éducation alimentaire développant l'esprit critique et l'envie de comprendre, et d'inscrire dès le plus jeune âge des routines comportementales protectrices comme le repas pris à heures fixes et partagé en famille. 


\section{Limites et pistes de recherche}

Cette recherche a étudié la perception des risques sanitaire et nutritionnel pour cinq types d'aliments, provenant de catégories différentes et présentant des caractéristiques variées. Il faudrait en tester un plus grand nombre et notamment intégrer plus de produits non ou peu transformés au-delà des pommes considérées ici. Il serait intéressant de comparer la PRS et la PRN du poisson, réputé pour ses qualités nutritionnelles mais comportant un risque de contamination par métaux lourds ; ou celles du fromage au lait cru, riche en calcium mais gras et salé, et plus susceptible d'être contaminé par la listeria. De plus, il serait utile de prendre en compte l'état de santé des individus. On peut s'attendre à ce que la PRS manifestée par les patients diabétiques soit plus élevée puisque leur alimentation influe directement sur leur qualité de vie.

Cette recherche montre aussi que la PRS et la PRN expliquent en partie l'intention d'achat, mais dans des proportions limitées. Pour vérifier si cela est dû à l'importance de facteurs tels que «la saveur et la sociabilité » encore très présentes dans le modèle culturel français (Ferrières 2006), on pourrait comparer les perceptions de risque alimentaire selon les quatre grands profils de consommateurs identifiés par Chalamon et Nabec (2013) : les optimisateurs (adeptes du naturel ou sportifs), les régulateurs (préventifs ou soucieux de leur ligne), les insouciants (gourmets ou gourmands), et les non-impliqués (mangeurs discount ou mangeurs pratiques). On peut penser que ces deux dernières catégories sont moins concernées par les aspects nutritionnels et sanitaires.

Enfin, les applications digitales nutritionnelles nécessitent des recherches pour évaluer si ces outils améliorent réellement les comportements alimentaires et chez quelles catégories de consommateurs. De plus, il faudrait s'assurer qu'elles ne génèrent pas de nouvelles peurs ou des comportements nocifs. D'après des entretiens préliminaires, certains les plébiscitent comme étant «géniales pour la santé (...) et indispensables quand on fait ses courses pour sélectionner les meilleurs produits» (Sébastien, 43 ans), quand d'autres les voient comme « source de stress : j'ai scanné tout ce que j'avais chez moi, rien n'allait, du coup j'ai laissé tomber »(Aline, 56 ans). Il faudrait aussi étudier si ces applications conduisent vraiment les entreprises de l'industrie agroalimentaire à améliorer le profil nutritionnel de leurs produits. 


\section{Références}

Argo, Jennifer J., Darren W. Dahl, et Andrea C. Morales. 2006. «Consumer Contamination: How Consumers React to Products Touched by Others ». Journal of Marketing 70 (2): 81-94.

Aurier, Philippe, et Lucie Sirieix. 2009. Marketing des produits agroalimentaires. Paris: Dunod.

Bauer, Raymond. 1960. «Consumer behavior as risk taking ». In Dynamic marketing for a changing world, proceedings of the 43rd conference of the AMA, 389-98. Chicago: American Marketing Association.

Bergadaà, Michelle, et Bertrand Urien. 2006. «Le risque alimentaire perçu comme risque vital de consommation: émergences, adaptation et gestion ». Revue Française de Gestion 32 (162): 127-44.

Brunel, Olivier, et Paul-Emmanuel Pichon. 2004. «Food-related risk-reduction strategies : purchasing and consumption processes ». Journal of Consumer Behaviour 3 (4): 360-74.

Chalamon, Isabelle, et Lydiane Nabec. 2013. «Les pratiques de lecture des étiquettes nutritionnelles: une analyse sémiotique des représentations des règles de nutrition ». Décisions marketing, $\mathrm{n}^{\mathrm{o}}$ 70: 59-74.

Cunningham, Scott M. 1967. «The major dimensions of perceived risk ». In Risk taking and information handling in consumer behaviour, par Donald F. Cox, 82-108. Boston: Harvard University Press.

Ferreira, Celio. 2006. «Food information environments: risk communication and advertising imagery ». Journal of Risk Research 9 (8): 851-68.

Ferrières, Madeleine. 2006. Histoire des peurs alimentaires : du Moyen Age à l'aube du XXe siècle. Paris: Seuil.

Fischler, Claude. 2001. «La peur est dans 1'assiette». Revue Française du Marketing 183/184 (3/4): 7-10.

Gallen, Céline. 2001. «Le besoin de réassurance en consommation alimentaire ». Revue Française du Marketing 183/184 (3/4): 67-85.

- 2005. «Le rôle des représentations mentales dans le processus de choix, une approche pluridisciplinaire appliquée au cas des produits alimentaires ». Recherche et Applications en Marketing 20 (3): 59-76.

Gallen, Céline, et Anne-Sophie Cases. 2007. « Le rôle du risque perçu et de l'expérience dans l'achat de vin en ligne ». Décisions Marketing, ${ }^{\circ} 45$ (janvier): 59-74. 
Gallen, Céline, Gaëlle Pantin-Sohier, et Dominique Peyrat-Guillard. 2019. «Les mécanismes cognitifs d'acceptation d'une innovation alimentaire de discontinuité: le cas des insectes en France ». Recherche et Applications en Marketing 34 (1): 50-77.

Gomez, Pierrick. 2008. «La nutrition dans les stratégies d'innovation alimentaire: de la protection du risque de santé publique à la construction de l'avantage concurrentiel ». Décisions Marketing, $\mathrm{n}^{\circ} 49$ (janvier): 71-83.

Guichard, Nathalie, et Isabelle Muratore. 2011. « Ce produit est-il sain? Trois approches pour appréhender le caractère sain d'un produit alimentaire à partir de son packaging ». Décisions marketing, $\mathrm{n}^{\mathrm{o}}$ 61: 55-66.

Jousse, Georges. 2004. Le risque, cet inconnu. Paris: Imestra.

Kasperson, Roger E., Paul Slovic, Ortwinn Renn, Halina Brown, Jacque Emel, Robert Goble, Jeanne Kasperson, et Samuel Ratick. 1988. "The social amplification of risk: a conceptual framework ». Risk Analysis: An International Journal 8 (2): 177-87.

Laporte, Marie-Eve, Géraldine Michel, et Sophie Rieunier. 2015. «Mieux comprendre les comportements alimentaires grâce au concept de perception du risque nutritionnel ». Recherche et Applications en Marketing 30 (1): 81-117.

Larceneux, Fabrice. 2003. «Segmentation des signes de qualité : labels expérientiels et labels techniques ». Décisions Marketing, $\mathrm{n}^{\circ} 29$ (mars): 35-46.

Mérigot, Philippe, et Lydiane Nabec. 2016. «Les effets d'alerte et de promotion des logos nutritionnels sur la face-avant des produits agroalimentaires ». Décisions Marketing, $\mathrm{n}^{\mathrm{o}}$ 83 (juillet): 29-47.

Nabec, Lydiane. 2017. «Améliorer les comportements alimentaires avec l'étiquetage nutritionnel : vers un agenda de recherche au service du Bien-être des consommateurs ». Recherche et Applications en Marketing 32 (2): 76-104.

Pichon, Paul-Emmanuel. 2012. «Réducteurs de risque». In Dictionnaire des cultures alimentaires, par Jean-Pierre Poulain, 1111-18. Presses Universitaires de France.

Rozin, Paul. 2005. «The meaning of food in our lives: a cross-cultural perspective on eating and well-being ». Journal of Nutrition Education \& Behavior 37 (37): 107-12.

Slovic, Paul. 1987. «Perception of risk ». Science 236 (4799): 280-85.

Thaler, Richard H., et Cass R. Sunstein. 2008. Nudge: Improving Decisions about Health, Wealth, and Happiness. New York: Penguin Books (2009).

Volle, Pierre. 1995. «Le concept de risque perçu en psychologie du consommateur: antécédents et statut théorique ». Recherche et Applications en Marketing 10 (1): 39-56. 